Communication

\title{
$A b$ initio study of electronic properties of armchair graphene nanoribbons passivated with heavy metal elements
}

\author{
P. Narin ${ }^{\text {a,* }}$, J.M. All Abbas ${ }^{\mathrm{a}}$, G. Atmaca ${ }^{\mathrm{a}}$, E. Kutlu ${ }^{\mathrm{b}}$, S.B. Lisesivdin ${ }^{\mathrm{a}}$, E. Ozbay ${ }^{\mathrm{c}, \mathrm{d}, \mathrm{e}}$ \\ ${ }^{a}$ Department of Physics, Faculty of Science, Gazi University, Teknikokullar, 06500, Ankara, Turkey \\ ${ }^{\mathrm{b}}$ Department of Energy Systems Engineering, Faculty of Engineering and Natural Sciences, Ankara Yuldirum Beyazit University, 06010 Ankara, Turkey \\ ${ }^{\mathrm{c}}$ Nanotechnology Research Center, Bilkent University, Bilkent, 06800 Ankara, Turkey \\ ${ }^{\mathrm{d}}$ Department of Physics, Bilkent University, Bilkent, 06800 Ankara, Turkey \\ ${ }^{\mathrm{e}}$ Department of Electrical and Electronics Engineering, Bilkent University, Bilkent, 06800 Ankara, Turkey
}

\section{A R T I C L E I N F O}

Commjunicated by L. Brey

Keywords:

GNR

Passivation

Electronic properties

Ab initio

Heavy metals

\begin{abstract}
A B S T R A C T
In this study, electronic properties of graphene nanoribbons with armchair edges (AGNRs) have been investigated with Density Functional Theory (DFT). Effects of heavy metal (HM) elements, including Zinc (Zn), Cadmium (Cd) and Mercury ( $\mathrm{Hg}$ ) atoms on electronic behavior of AGNRs have been calculated by passivating for both one and two edges of AGNRs in detail. To explain the electronic behavior of investigated AGNRs, the electronic band structure, the density of states (DOS), total energy have been calculated. Energetically favorable structures have been determined using calculated binding energy values. The obtained bandgap values of investigated structures changes between 0.30 and $0.64 \mathrm{eV}$. Increasing atomic number of passivation atoms have led to an increment in the bandgap of AGNRs.
\end{abstract}

\section{Introduction}

Carbon-based materials have been attracted more attention of researchers over the last decade, due to their unique structural and electronic properties [1-6]. Graphene is one of them which attracted much attention since it is discovered experimentally in 2004. It is a monolayer honeycomb lattice of $\mathrm{sp}^{2}$ bonded carbon $[7,8]$. Because of its unique and exceptional electronic transport properties, graphene has the potential application in many fields such as nanoelectronics and renewable energy storage $[7,9]$.

Graphene nanoribbons (GNR) are the one-dimensional (1D) materials obtained from the graphene and have been appealed in great amount regards in the fields of materials science and physics $[1,10]$. According to the feature of edge, the GNRs are divided into zigzag edge graphene nanoribbons (ZGNRs) and armchair edge graphene nanoribbons (AGNRs) [11-13]. The GNRs have been studied theoretically and experimentally because of their unique electronic structures and transport properties [14]. The electronic structure of GNRs depends on their width and edge structure [15]. Generally, AGNRs show mostly semiconductor properties, ZGNRs have a metallic behavior as known. However, these properties of GNRs can be changed with doping, edge passivation, applied electric field perpendicular to nanoribbons [16-18]. As well as metallic and semiconductor properties of GNRs, spin-dependent structures can be obtained in these ways [19]. By this means, the electronic behavior of GNRs can be mostly controlled.

In recent years, graphene and carbon nanotubes (CNTs) have been experimentally used for detections of heavy metal (HM) elements [20]. Graphene-based sensors for such HM elements are useful even small concentrations of HM atom concentrations [21]. Therefore, detection of $\mathrm{HM}$ elements is important for many industrial applications, especially for medical applications. Despite a presence of interactions between graphene and HM elements, however, there is lack of numerical calculations about the effect of HM elements on the electronic properties of low dimensional carbon-based materials like nanoribbons.

In this study, effects of some HM elements used as passivation on electronic properties of AGNRs have been studied with density functional theory (DFT) method. Discusses on the structural stability and the electronic behavior of the each studied structures on calculating binding energy, electronic band structures, density of states (DOS) have been made.

\section{Calculation method}

In this work, we explore the electronic band structure and the DOS of asymmetrical AGNRs passivated with $\mathrm{H}$ and $\mathrm{HM}$ elements including Zn, Cd, and Hg. The calculations were carried out Atomistix Toolkit-

\footnotetext{
* Corresponding author

E-mail address: polatnarin0@gmail.com (P. Narin).
} 
Virtual NanoLab (ATK-VNL) software package based on DFT was used to perform the calculations in order to obtain the electronic properties of investigated asymmetrical AGNRs [22,23]. ATK can be used for the calculation of basic material properties such as electronic, structural and optical properties of atomic scale materials using Density Functional Theory. The used pseudopotentials are varied with the upgraded versions of the software. In our calculations, which are calculated with ATK 2017.2, SG15 Optimized Norm-Conserving Vanderbilt (ONCV) pseudopotentials [24] are used to perform high accuracy in the required calculations. As an exchange-correlation function, the spin-polarized version of the generalized gradient approximation (SGGA) was used in the form of Perdew, Burke and Ernzerhof (PBE) correlation functional [25]. An energy cut-off value of $500 \mathrm{eV}$ was selected to ensure accurate results. Brillouin zone integration was performed using a regular Monkhorst-Pack Scheme with $1 \times 1 \times 100$ k-point grid. All atoms were fully relaxed and maximum force components on all atoms were accepted to be less than $0.05 \mathrm{eVA}^{-1}$. The calculations for zigzag and armchair edges of GNRs were performed, but the results of zigzag edges GNR weren't presented due to their metallic electronic band structures. Also, the electronic calculations were carried out for different widths, however, nanoribbons with different widths show similar results with each other. The width of the investigated asymmetrical AGNR structures in this study was $\mathrm{N}=8$ as illustrated in Fig. 1 (a). In asymmetrical AGNR, the supercell is periodic along z-direction as shown in Fig. 1 (a). In our calculations, we modeled asymmetrical AGNRs with one and both edges passivated with $\mathrm{Zn}, \mathrm{Cd}$, and $\mathrm{Hg}$ so that they are compared with each other in terms of the structural stability, the electronic band structure, and the band gap. Structural stability of investigated structures was determined using Eq. (1).

$E_{b}=E_{T}-E_{T: \text { bare }}-n E_{H}-m E_{P}$

where, $E_{b}$ is binding energy, $E_{T}$, and $E_{T: b a r e}$, are total energies of the structure including $\mathrm{HM}$ elements and bare structure, $E_{H}$ is energy of isolated $\mathrm{H}, E_{P}$ is energy of isolated passivation atom, $n$ and $m$ are numbers of $\mathrm{H}$ and passivation atoms, respectively.

\section{Results and discussions}

The atomic configuration and the electronic band structure of the AGNR passivated with $\mathrm{H}$ atoms have been shown in Fig. 1. The electronic band structure and DOS of asymmetrical AGNR show a semiconductor behavior as expected. Fermi level is in the middle of conduction and valence bands and represented with a red line. The bond length of AGNR passivated with $\mathrm{H}$ atoms has been found as $1.10 \AA$, the calculated binding energy and the electronic band gap has been obtained as 0.85 and $0.46 \mathrm{eV}$, respectively. Previously, the calculated
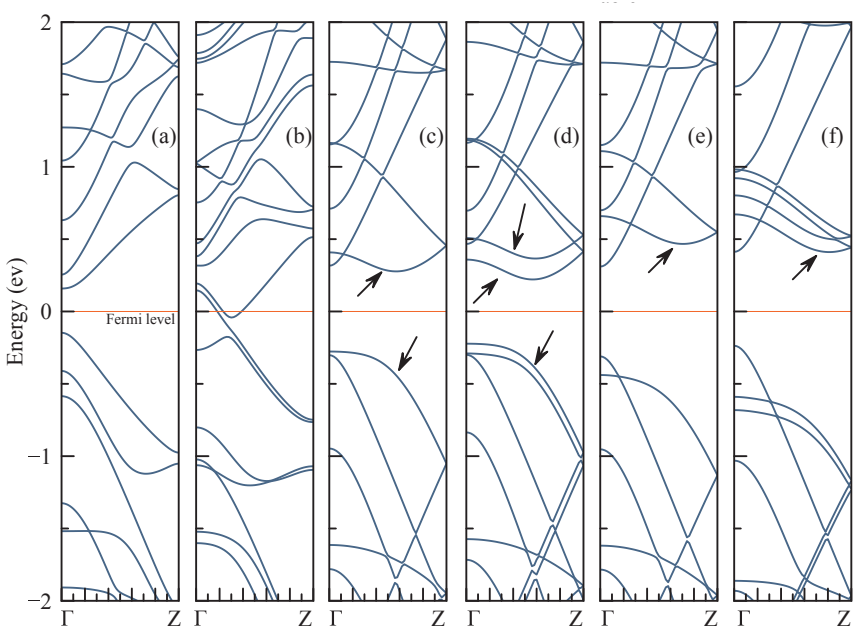

Fig. 2. Band structures of AGNR passivated with HM elements. (a), (c), (e) are represented one edge passivated structures for $\mathrm{Zn}, \mathrm{Cd}, \mathrm{Hg}$, respectively. (b), (d), (f) are shown passivated structures on both edges with $\mathrm{Zn}, \mathrm{Cd}, \mathrm{Hg}$, respectively.

energy band gaps for AGNR passivated with $\mathrm{H}$ atoms are found to be give similar results with this study [26,27].

Fig. 2 shows the electronic band structures of AGNRs passivated with the investigated HM elements. While Fig. 2a), c) and e) figures show one edge passivated structures, others represent passivated structures on both edges. All investigated AGNRs have a semiconductor behavior except the structure passivated with $\mathrm{Zn}$ on both edges. While AGNRs passivated with $\mathrm{Zn}$ and $\mathrm{Hg}$ atoms have observed a direct bandgap, the structure passivated with $\mathrm{Cd}$ atom has an indirect bandgap. In the investigated structures, any spin polarization has not been observed. This shows that the AGNRs passivated with HM elements have still a magnetization symmetry, however, previously some studies present that when non-metal Aluminum (Al) and metallic Iron (Fe) atoms are adsorbed on the edges of ZGNRs, these atoms generate the spin polarized ZGNRs [28,29]. These results have been explained with breaking of the magnetization symmetry in ZGNRs. The source of the spin polarization inducing is deterioration in edge states of GNRs by adsorbed or passivated atoms. It is shown that the impurity bands marked by arrows for some band structures are formed due to hybridization of $\pi$-orbitals of $\mathrm{C}$ atoms and $\mathrm{d}$ and $\mathrm{f}$ orbitals of passivation atoms, compared with the electronic band structure pure AGNR. For the structures passivated on both edges, a numerous of band splittings are observed due to overlap of band states related to each similar passivated edge. The bandgap of the structures is higher as the atomic
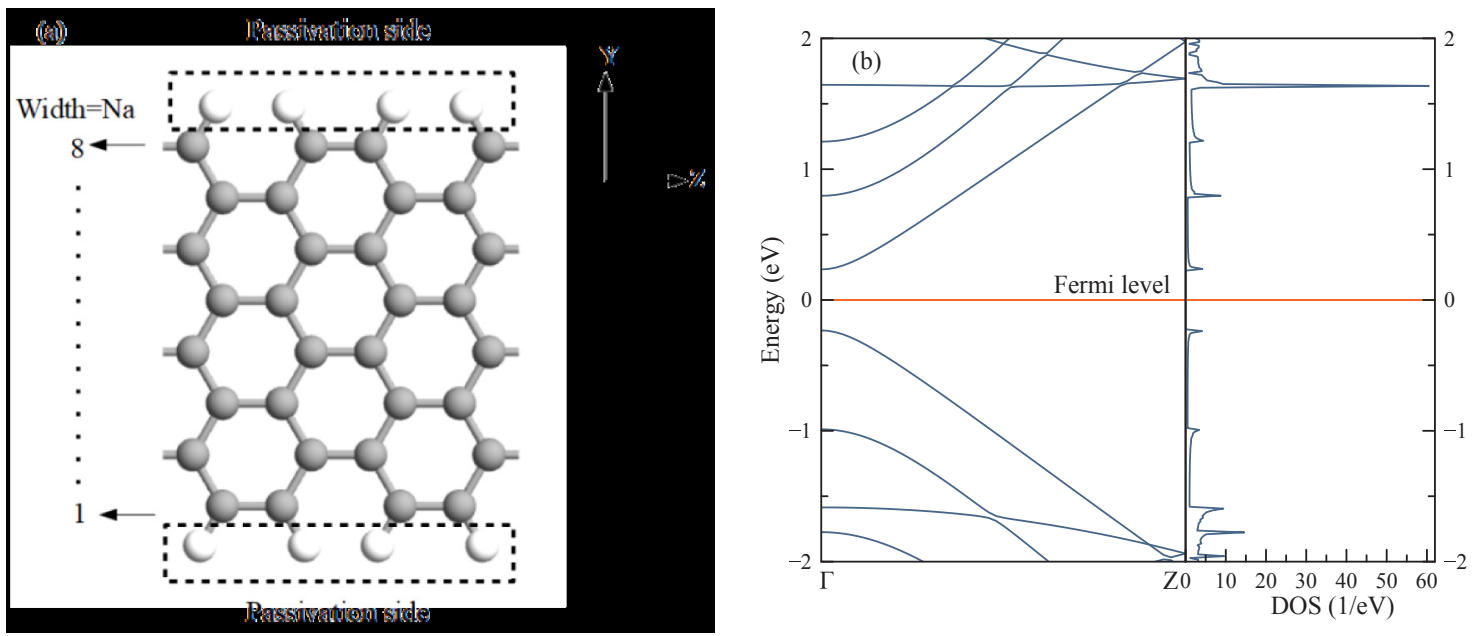

Fig. 1. a) Atomic configuration of used AGNRs b) electronic band structure and DOS passivated AGNR with Hydrogen atoms. 
Table 1

Calculated electronic and structural parameters for AGNRs with the investigated HM elements.

\begin{tabular}{lllll}
\hline Structure & $\begin{array}{l}\mathrm{E}_{\mathrm{b}}(\mathrm{eV}) \text { per } \\
\text { atom }\end{array}$ & $\mathrm{E}_{\text {gap }}(\mathrm{eV})$ & $\begin{array}{l}\text { Type of } \\
\text { bandgap }\end{array}$ & $\begin{array}{l}\text { Bond length of C- } \\
\mathrm{HM}(\AA)\end{array}$ \\
\hline One edge $\mathrm{Zn}$ & -0.69 & 0.30 & Direct & 1.87 \\
Both edges $\mathrm{Zn}$ & -0.03 & 0 & Metallic & 1.68 \\
One edge Cd & -0.52 & 0.55 & Indirect & 2.33 \\
Both edges Cd & -0.19 & 0.43 & Indirect & 2.32 \\
One edge Hg & -0.45 & 0.62 & Direct & 2.35 \\
Both edges Hg & 0.44 & 0.64 & Direct & 2.43
\end{tabular}

number of the investigated HM elements is increased. Moreover, one edge passivated AGNRs have a higher bandgap than both edges passivated structures due to overlap of band states related to each similar passivated edge. The calculated bandgap values are listed in Table I.

DOS of the investigated structures are shown in Fig. 3. Similar to adsorption studies, there is no spin-dependent result in AGNR structure passivated with $\mathrm{Zn}, \mathrm{Cd}$ and $\mathrm{Hg}$ [30]. In pure AGNR, one dimensional (1D) DOS behavior is shown from Fig. 1b). Investigated AGNR structures with HM elements indicate quasi one-dimensional (Q1D) and quasi zero-dimensional (Q0D) local density of state behaviours [11]. Observing a Q0D behavior in these structures is expected because of a highly localized $\mathrm{d}$ and $\mathrm{f}$ orbitals of For the structures passivated on both edges, a numerous of band splittings are observed due to overlap of band states related to each similar passivated edge passivation atoms. Q1D behavior can be associated to nanowire-like bonding of HM elements at the edge of the investigated AGNRs.

Fig. 4 shows changing of the bond length between Carbon (C) and investigated HM elements and energy band gaps in passivated AGNR

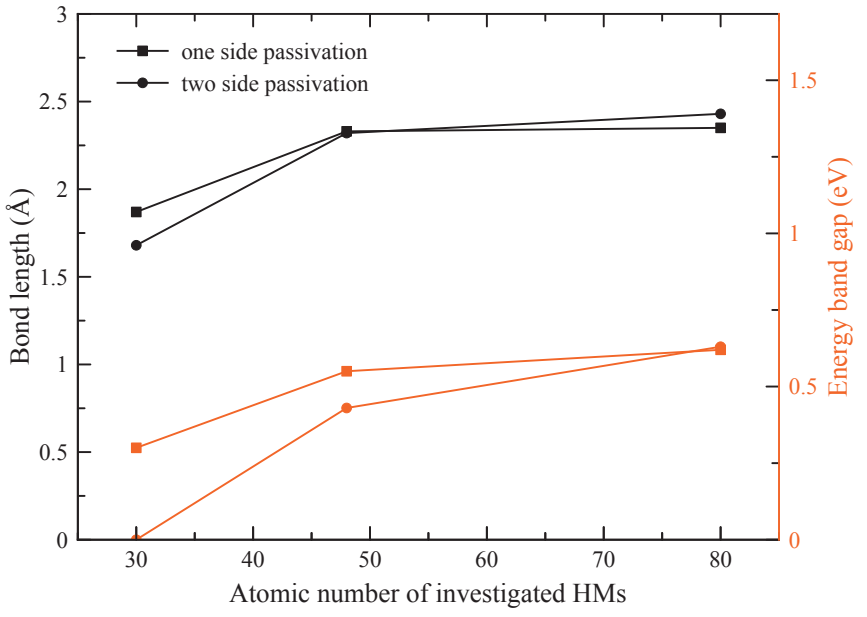

Fig. 4. Bond length and energy bandgap of investigated structures depending on the atomic number of the investigated HM elements.

with respect to the atomic number of the investigated HM elements. In accordance with the results reported in the literature, the bond length is increased with the increasing of atomic number of $\mathrm{HM}$ elements as $1.73 \AA, 2.22 \AA$ and $2.28 \AA$ for $\mathrm{C}-\mathrm{Zn}, \mathrm{C}-\mathrm{Cd}$ and $\mathrm{C}-\mathrm{Hg}$ bond lengths, respectively $[21,31]$. Moreover, the band gap values seen at the electronic band structures given in Fig. 2 are increased with increasing atomic number of the investigated HM elements. It is shown that small strain distributions could be changed the band gap of AGNRs [32]. In our case, passivated AGNRs with high atomic number atoms had small local strain distributions at the edge of nanoribbons. This situation is
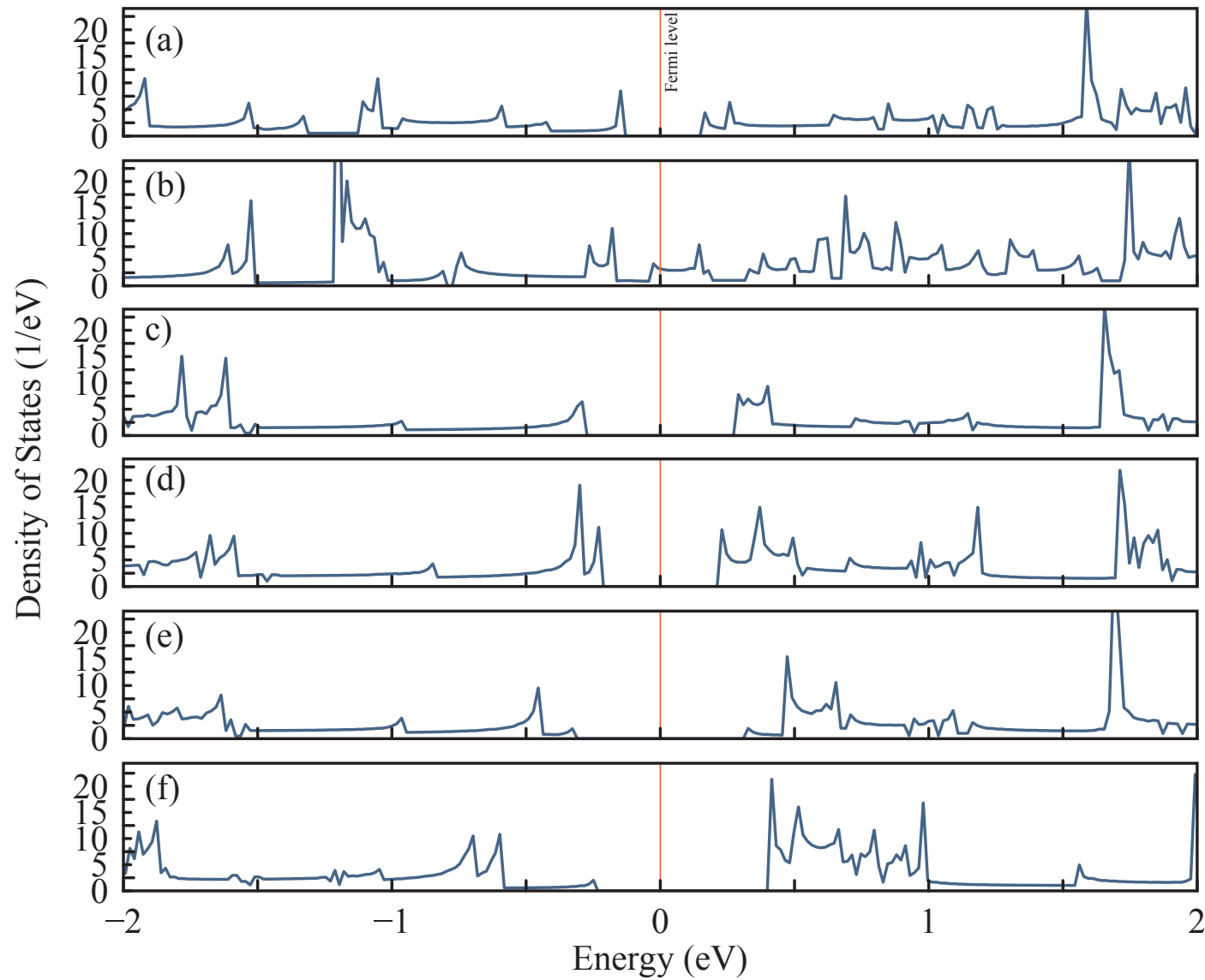

Fig. 3. Density of States of AGNR passivated with $\mathrm{Zn}, \mathrm{Cd}$ and $\mathrm{Hg}$ atoms (a), (c), (e) are represented one edge passivated structures for $\mathrm{Zn}, \mathrm{Cd}, \mathrm{Hg}$, respectively (b), (d), (f) are shown both edges passivated structures for $\mathrm{Zn}, \mathrm{Cd}, \mathrm{Hg}$, respectively. 
expected to lead a decrease in the band gap.

According to the $E_{b}$ results listed in Table I, the structural stability of the passivated AGNRs can be interpreted. It can be seen that the structure passivated with $\mathrm{Zn}$ one edge is the most stable structure after AGNR passivated with $\mathrm{H}$ atom. It is reported that GNRs passivated with transition metals are found to be more stable than both edges passivated structures [33-35].

\section{Conclusion}

In this study, the investigations of effects of some HM elements as passivation atoms on electronic structures of AGNRs have been studied by means of DFT method. The bandgap of investigated structures is changed from 0.30 to $0.64 \mathrm{eV}$ along with the investigated HM elements. With the increase of the atomic number of HM elements, the band gap values of AGNRs have been increased. Unlike other investigated structures, AGNR passivated with Cd atom shows indirect band gap. Q0D and Q1D type some local DOS behaviors have been shown in the DOS results of AGNRs passivated with the investigated HM elements. The most stable structure is determined as AGNR one edge passivated with $\mathrm{Zn}$ among the studied structures with the investigated HM elements interpreting binding energy results for each structure. These results are important in terms of understanding of the effect of HM elements on electronic properties of low dimensional carbon-based materials like graphene nanoribbons.

\section{Acknowledgments}

This work was supported by TUBITAK under Project No. 116F197. E. O. acknowledges partial support from the Turkish Academy of Sciences. S. B. L was supported in part by the Distinguished Young Scientist Award of Turkish Academy of Sciences (TUBA-GEBIP 2016).

\section{References}

[1] S.L. Chang, S.Y. Lin, S.K. Lin, C.H. Lee, M.F. Lin, Sci. Rep. 4 (2014) 1-8.

[2] A.K. Geim, Graphene: status and prospects, Science 324 (5934) (2009) 1530-1534.

[3] X. Li, X. Wang, L. Zhang, S. Lee, H. Dai, Chemically derived, ultrasmooth graphene nanoribbon semiconductors, Science 319 (2008) 1229-1232.

[4] D.V. Kosynkin, A.L. Higginbotham, A. Sinitskii, J.R. Lomeda, A. Dimiev, B.K. Price, J.M. Tour, Longitudinal unzipping of carbon nanotubes to form graphene nanoribbons, Nature 458 (2009) 872-876.

[5] F. Cataldo, G. Compagnini, G. Patané, O. Ursini, G. Angelini, P.R. Ribic, G. Margaritondo, A. Cricenti, G. Palleschi, F. Valentini, Graphene nanoribbons produced by the oxidative unzipping of single-wall carbon nanotubes, Carbon 48 (2010) 2596-2602.

[6] D.B. Shinde, M. Majumder, V.K. Pillai, Counter-ion dependent, longitudinal unzipping of multi-walled carbon nanotubes to highly conductive and transparent graphene nanoribbons, Sci. Rep. 4 (2014) 1-6.

[7] M.J. Allen, V.C. Tung, R.B. Kaner, Honeycomb carbon: a review of graphene, Chem. Rev. 110 (2009) 132-145.

[8] W. Wang, G. Zhao, First principles study of the electronic energy bands and state density of lithium-doped narrow armchair graphene nanoribbons, Solid State Commun. 166 (2013) 6-11.

[9] S.H. Tan, L.M. Tang, K.Q. Chen, Band gap opening in zigzag graphene nanoribbon modulated with magnetic atoms, Curr. Appl. Phys. 14 (2014) 1509-1513.

[10] W.H. Liao, B.H. Zhou, H.Y. Wang, et al., Electronic structures for armchair-edge graphene nanoribbons under a small uniaxial strain, Eur. Phys. J. B 76 (2010) $463-467$.

[11] B. Sarikavak-Lisesivdin, S.B. Lisesivdin, E. Ozbay, Ab initio study of Ru-terminated and Ru-doped armchair graphene nanoribbons, Mol. Phys. 110 (2012) 2295-2300.

[12] A.F. Kuloglu, B. Sarikavak-Lisesivdin, S.B. Lisesivdin, E. Ozbay, First-principles calculations of Pd-terminated symmetrical armchair graphene nanoribbons, Comput. Mater. Sci. 68 (2013) 18-22.

[13] P. Narin, E. Kutlu, B. Sarikavak-Lisesivdin, S.B. Lisesivdin, E. Ozbay, Electronic properties of Li-doped zigzag graphene nanoribbons, Physica E 84 (2016) 543-547.

[14] Q. Yan, B. Huang, J. Yu, Z. Fawei, J. Zang, J. Wu, B.L. Gu, F. Liu, W. Duan, Intrinsic current - voltage characteristics of graphene nanoribbon transistors and effect of edge doping, Nano Lett. 7 (2007) 1469-1473.

[15] H. Raza, E.C. Kan, Armchair graphene nanoribbons: electronic structure and electric-field modulation, Phys. Rev. B 77 (2008) 1-5.

[16] Y.W. Son, M.L. Cohen, S.G. Louie, Half-metallic graphene nanoribbons, Nature 444 (2006) 347-349.

[17] V. Barone, O. Hod, G.E. Scuseria, Electronic structure and stability of semiconducting graphene nanoribbons, Nano Lett. 6 (2006) 2748-2754.

[18] Y. Li, Z. Zhou, P. Shen, Z. Chen, Spin gapless semiconductor - metal - half-metal properties in nitrogen-doped zigzag graphene nanoribbons, ACS Nano 3 (2009) 1952-1958.

[19] E. Cruz-Silva, Z.M. Barnett, B.G. Sumpter, V. Meunier, Structural, magnetic, and transport properties of substitutionally doped graphene nanoribbons from first principles, Phys. Rev. B 83 (83) (2011) 1-9.

[20] H. Huang, T. Chen, X. Liu, H. Ma, Ultrasensitive and simultaneous detection of heavy metal ions based on three-dimensional graphene-carbon nanotubes hybrid electrode materials, Anal. Chim. Acta 852 (2014) 45-54.

[21] I. Shtepliuk, N.M. Caffrey, T. Iakimov, V. Khranovskyy, I.A. Abrikosov, R. Yakimova, On the interaction of toxic Heavy Metals $(\mathrm{Cd}, \mathrm{Hg}, \mathrm{Pb})$ with graphene quantum dots and infinite graphene, Sci. Rep. 7 (2017) 1-17.

[22] Version 2017.2 QuantumWise A/S, (2017) http://www.quantumwise.com.

[23] E. Artacho, E. Anglada, O. Diéguez, J.D. Gale, A. García, J. Junquera, R.M. Martin, P. Ordejón, J.M. Pruneda, D. Sánchez-Portal, J.M. Soler, The SIESTA method; developments and applicability, J. Phys. Condens. Matter 20 (2008) 1-6.

[24] D.R. Hamann, Optimized norm-conserving Vanderbilt pseudopotentials, Phys. Rev. B 88 (2013) 085117.

[25] J.P. Perdew, K. Burke, M. Ernzerhof, Generalized gradient approximation made simple, Phys. Rev. Lett. 77 (1996) 3865-3868.

[26] Z. Liu-Jun, X. Tong-Sheng, The complex band structure for armchair graphene nanoribbons, Chin. Phys. B 19 (2010) 117105.

[27] U. Treske, F. Ortmann, B. Oetzel, K. Hannewald, F. Bechstedt, Electronic and transport properties of graphene nanoribbons, Phys. Status Solidi 207 (2010) 304-308.

[28] Z.L. Zhang, Y.P. Chen, Y.E. Xie, M. Zhang, J.X. Zhong, Spin-polarized transport properties of Fe atomic chain adsorbed on zigzag graphene nanoribbons, J. Phys. D Appl. Phys. 44 (2011) 215403

[29] Y.Q. Wang, Y.E. Xie, Z.L. Zhang, Y. Zhang, Y.P. Chen, Spin-polarized transport in zigzag graphene nanoribbons adsorbing nonmagnetic atomic chain, Eur. Phys. J. B 86 (2013) 34.

[30] I.A. Pašti, A. Jovanović, A.S. Dobrota, S.V. Mentus, B. Johansson, N.V. Skorodumova, Atomic adsorption on pristine graphene along the Periodic Table of Elements-From PBE to non-local functionals, Appl. Surf. Sci. 436 (2018) $433-440$

[31] H.P. Zhang, X.G. Luo, X.Y. Lin, X. Lu, Y. Leng, Density functional theory calculations of hydrogen adsorption on Ti-, Zn-, Zr-, Al-, and N-doped and intrinsic graphene sheets, Int. J. Hydrogen Energy 38 (2013) 14269-14275.

[32] F. Ma, Z. Guo, K. Xu, P.K. Chu, First-principle study of energy band structure of armchair graphene nanoribbons, Solid State Commun. 152 (2012) 1089-1093.

[33] N.K. Jaiswal, P. Srivastava, Structural stability and electronic properties of Nidoped armchair graphene nanoribbons, Solid State Commun. 151 (2011) 1490-1495.

[34] N. Tyagi, N.K. Jaiswal, K.K. Jha, V. Sharma, P. Srivastava, Structural, magnetic and electronic properties of armchair graphene nanoribbons interacting with Co: DFT investigations, Ferroelectrics 519 (2017) 178-186.

[35] M. Kumar, N.K. Puri, N. Tyagi, P. Srivastava, Band gap engineering of armchair graphene nanoribbons via Mn/Cr termination, J. Comput. Theor. Nanosci. 14 (5) (2017) 2401-2404. 